\title{
Fabrication of a Transparent Anti-stain Thin Film Using an Atmospheric Pressure Cold Plasma Deposition System
}

\author{
Y. Suzaki ${ }^{1}$, K. Yamauchi ${ }^{1}$, H. Miyagawa ${ }^{1}$, K. Yamaguchi ${ }^{1}$, T. Shikama ${ }^{2}$, and K. Ogawa ${ }^{1}$ \\ ${ }^{1}$ Kagawa University, 2217-20 Hayashi-cho, Takamatsu, Kagawa 761-0396, Japan \\ ${ }^{2}$ Kagawa National College of Technology, Chokushi-cho, Takamatsu, Kagawa 761-8058, Japan
}

\begin{abstract}
Recently, outdoor-constructed solar panels have a problem such as power generation efficiency is reduced by the face plate dirt. On the other hand, electronic touch panels have a problem such as deterioration of visibility of the screen by finger grease stain. To solve these problems, we need to fabricate the anti-stain surfaces which have superhydrophobic and oil-repellent abilities without spoiling the transparency of the transparent substrate. In this study, we fabricated lotus leaves like surface on a glass substrate. Firstly, $\mathrm{SiO}_{2}$ particles of ca. $100 \mathrm{~nm}$ diameter were arranged on the glass substrates. Secondly, to obtain the fractal-like structure (ultra-micro-rough structure) on the surface, $\mathrm{ZnO}$ thin film having a columnar structure was fabricated on the $\mathrm{SiO}_{2}$ particles by using an atmospheric pressure cold plasma deposition system. By using these processes, the $\mathrm{ZnO}$ columns formed radiantly on the spherical surface of the $\mathrm{SiO}_{2}$ particles. Furthermore, without spoiling the ultra-micro-rough structure, a transparent anti-stain monolayer with low surface energy was prepared by using a chemical adsorption technique onto the surface. Average value of the water droplet contact angles of the samples fabricated was $151.8 \mathrm{deg}$. Field emission scanning electron microscope (FESEM) observation reviled that this sample has a raspberry structure in which columnar structure has grown radially on the $\mathrm{SiO}_{2}$ particles.
\end{abstract}

\section{Introduction}

One of major problems with outdoor-constructed solar panels is the reduction of the efficiency of power generation caused by the surface stain. On the other hand, a problem with electronic touch screens is the deterioration of screen visibility caused by finger grease stains. To solve these problems, we need to fabricate transparent anti-stain surfaces, which have superhydrophobicity, and which is defined as having more than $150 \mathrm{deg}$. water drop contact angles, and oilrepellency. These functions can be realized by preparing a microscopic fractal structure (ultra-micro-rough structure) like the surface of a lotus leaf, because such fractal structure is considered to have both the "lotus effect" [1] and the "easy oil wiping."

Wenzel [2] reported that uneven surfaces can increase water repellency, because the surface area is larger than flat surface. In his paper, the flat surface is thought to have the limitation of water-repellency with the maximum value of the water drop contact angle as 120 deg. [3] This means that we cannot realize the superhydrophobicity as long as we use flat surfaces. In this paper, we aim to fabricate fractal-like surface like lotus leaf on glass substrates and to produce a superhydrophobic surface.

\section{Sample preparation}

Three processes were used to prepare the samples of the transparent anti-stain film. Firstly, $\mathrm{SiO}_{2}$ particles of about $100 \mathrm{~nm}$ diameters in average were arranged on glass substrates. Secondly, the thin zinc-oxide film with a columnar structure was fabricated on the $\mathrm{SiO}_{2}$ particles using an atmospheric pressure cold plasma deposition system.

It is reported [4] that when a thin $\mathrm{ZnO}$ film was deposited on flat glass substrate, the $\mathrm{ZnO}$ film was formed with packed sharp columns with about $30 \mathrm{~nm}$ width and $300 \mathrm{~nm}$ lengths, which are standing vertical to the surface plane. It was expect that when $\mathrm{ZnO}$ is deposited on the spherical surface of $\mathrm{SiO}_{2}$ particle, $\mathrm{ZnO}$ grows radiantly on the particle. Afterwards, the $\mathrm{ZnO}$ column pillars formed on the spherical surface of the $\mathrm{SiO}_{2}$ particles, that we call raspberry structure, as shown in Fig. 1.

Moreover the raspberry structure was covered with chemically adsorbed monomolecular layer (CAM), which enhances anti-stain property. Details of each process are described as follows. 


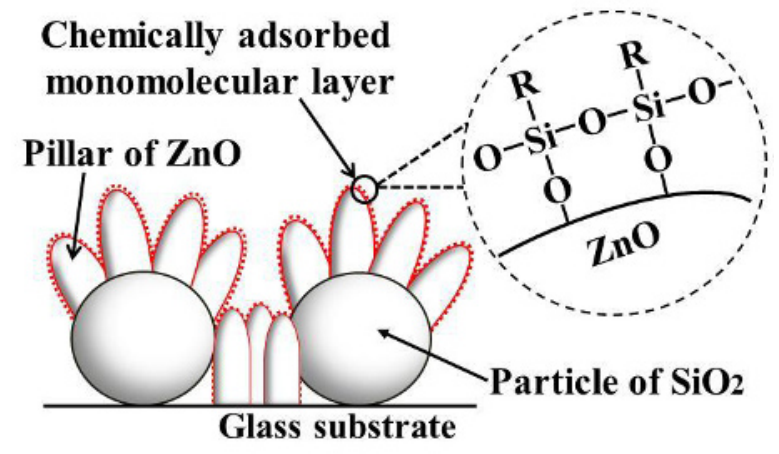

Fig. 1. Raspberry structure was made by transparent silica particles of $100 \mathrm{~nm}$ in diameter, and transparent columnar structure of Zinc oxide, and ultra- hydrophobicity can be realized by preparing a hydrophobic monomolecular layer without spoiling the roughness. " $R$ " means the fluorocarbon group and/or alkyl group.

\subsection{Arrangement of $\mathrm{SiO}_{2}$ particles}

In advance, a glass substrate was treated by corona discharge (applied $\mathrm{rf}$ power; $250 \mathrm{~W}$, treatment time; 1 min) to remove surface dirt and to get surface energy of ca. $70 \mathrm{mN} / \mathrm{m}$. After the treatment, $\mathrm{SiO}_{2}$ particles were arranged on the substrate surface by a dip coating technique: where the substrate was dipped in the $\mathrm{SiO}_{2}$ particle suspension, and pulled out at a constant rate, as shown in Fig. 2. In this technique, one can easily control the density of $\mathrm{SiO}_{2}$ particles, i.e., when the pulling speed is fast (slow), the density on the substrate becomes dense (sparse). The $\mathrm{SiO}_{2}$ particle suspension was made of the mixture of ethanol (99.5\% purity, $34 \mathrm{ml})$, COLCOAT P $(6 \mathrm{ml})$ and IPA-ST-ZL $(20 \mathrm{ml})$. Average size of $\mathrm{SiO}_{2}$ particle in IPA-ST-ZL is $100 \mathrm{~nm}$.

Several types of samples were made with different pulling out speeds $(0.23 \mathrm{~mm} / \mathrm{s}, 0.1 \mathrm{~mm} / \mathrm{s}$ and $0.05 \mathrm{~mm} / \mathrm{s})$ and the substrates were sintered in an electric furnace at temperature of $500{ }^{\circ} \mathrm{C}$. The density of silica particles were 34,46 and 86 particles $/ \mu \mathrm{m}^{2}$ when the pulling out speeds were $0.05,0.1$ and $0.23 \mathrm{~mm} / \mathrm{s}$, respectively.

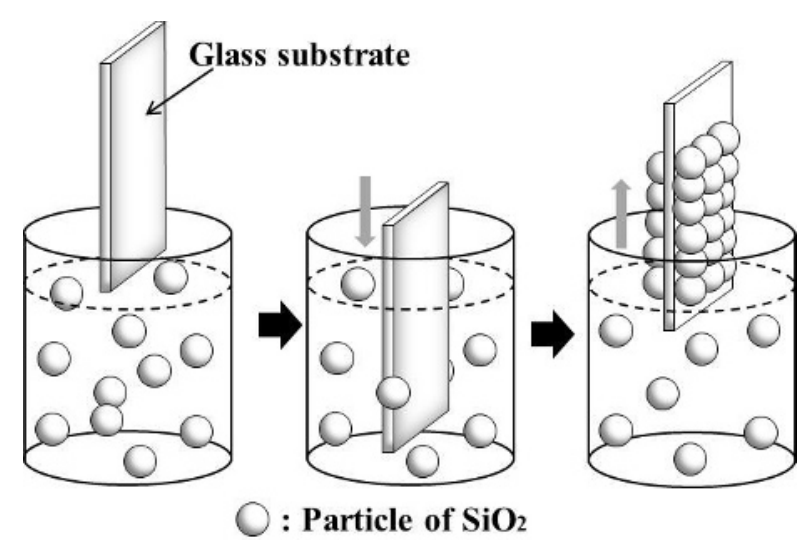

Fig. 2. Dip coating technique to fabricate the arrangement of $\mathrm{SiO}_{2}$ particles. The substrate was dipped in the $\mathrm{SiO}_{2}$ particle suspension, and pulled out at constant speed.
And then, the most suitable speed was determined to be $0.23 \mathrm{~mm} / \mathrm{s}$ for the closed packing arrangement of the $\mathrm{SiO}_{2}$ particles, wherein pencil hardness was $2 \mathrm{H}$.

\subsection{Deposition of $\mathrm{ZnO}$ thin films}

The $\mathrm{ZnO}$ thin films were deposited on the $\mathrm{SiO}_{2}$ particles arranged substrates with an atmospheric pressure cold plasma (APCP) deposition system. Figure 3 shows a schematic layout of the APCP system. In Fig. 3 , Oxygen gas and helium gas containing raw material is introduced into a torch of glow discharge plasma generated by pulsed voltage of $1.0 \mathrm{kV}$. As the raw material for the $\mathrm{ZnO}$ deposition, 2-methoxy-6-methyl3,5-heptanedionato was used (Zn-MOPD, Ube Industries, Ltd.). The molecular structure of Zn-MOPD is shown in Fig. 4. Zn-MOPD is decomposed in plasma torch and $\mathrm{ZnO}$ is deposited on the substrate surface.

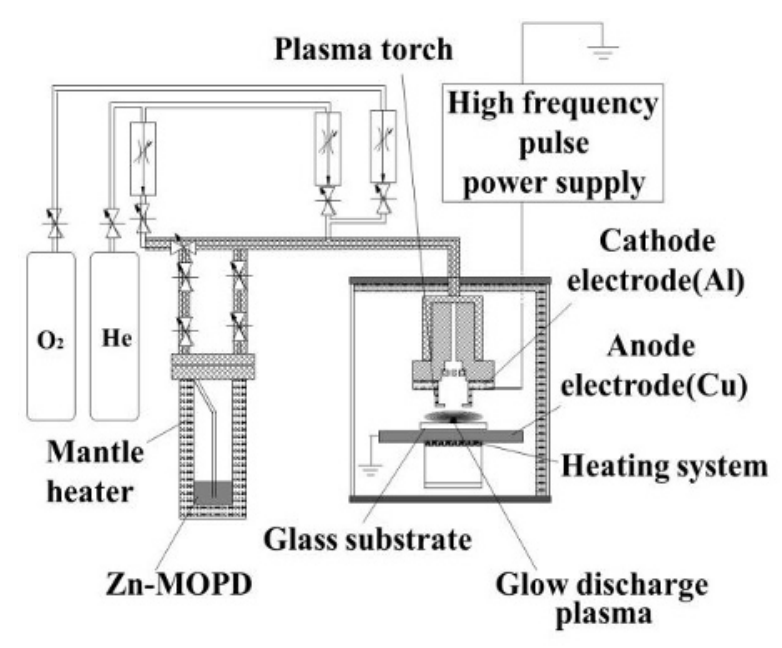

Fig. 3. Schematic layout of our atmospheric pressure cold plasma (APCP) deposition system. Helium gas containing ZnMOPD is introduced into a plasma torch. Oxygen gas was used in order to fabricate the thin $\mathrm{ZnO}$ films with this APCP deposition system.

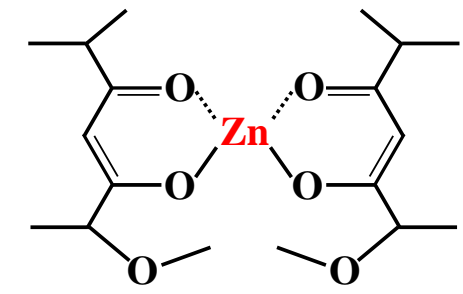

Fig. 4. The molecular structure of Zn-MOPD. Zn-MOPD is a yellow liquid reagent at room temperature, and molecular weight is 407.8. In this research, the vaporized $\mathrm{Zn}$-MOPD which heated to $100{ }^{\circ} \mathrm{C}$ by the electric mantle heater was introduced with helium.

Table 1 shows the preparation conditions and water drop contact angles measured. The pulling out velocities in dipping process were $0.05 \sim 0.23 \mathrm{~mm} / \mathrm{s}$. The substrate temperature during the deposition was set at $215^{\circ} \mathrm{C}$ for 
sample A, and those of others were at $50{ }^{\circ} \mathrm{C}$. The water drop contact angles measured shows average value and $\mathrm{S}$. D. shows standard deviation of the contact angles.

Table 1. Growth conditions of the samples and results of water drop contact angles measured.

\begin{tabular}{|c|c|c|c|c|}
\hline Sample & $\begin{array}{c}\text { Pulling } \\
\text { out } \\
\text { velocity } \\
(\mathrm{mm} / \mathrm{s})\end{array}$ & $\begin{array}{c}\text { Substrate } \\
\text { temperature } \\
\left({ }^{\circ} \mathrm{C}\right)\end{array}$ & $\begin{array}{c}\text { Average } \\
\text { value of } \\
\text { contact } \\
\text { angles }\left({ }^{\circ}\right)\end{array}$ & S.D. \\
\hline $\mathrm{A}$ & 0.10 & 215 & 151.8 & 1.4 \\
\hline $\mathrm{B}$ & 0.23 & 50 & 143.6 & 0.4 \\
\hline $\mathrm{C}$ & 0.10 & 50 & 138.3 & 1.1 \\
\hline $\mathrm{D}$ & 0.05 & 50 & 143.7 & 1.3 \\
\hline
\end{tabular}

\subsection{Preparation of CAM}

After the $\mathrm{ZnO}$ deposition, the substrate was covered with CAM, where samples were dipped in the chemisorptions solution for one hour, pulled out, and dried in an air atmosphere during one day. The molecule((Heptadecafluoro-1,1,2,2-tetradecyl)

trimethoxysilane: FAS17) used for CAM has a terminal reactive site which is composed of the methoxy-silyl group and a functional site having the fluorocarbon group, as shown in Fig. 5. This CAM is effective to repel the water and oil. Because it's fluorocarbon group reduce the surface energy of the base substrate. In the chemisorption process, CAM is prepared self-assembly due to the chemical reaction between the reactive site of the molecule and the substrate surface. Firstly, methoxy-silyl groups of the reactive site change to the silanol groups by the dealcoholization reaction with the adsorbed water at the substrate surface. And then, the silanol groups react to the hydroxyl groups of the substrate surface [5]. In this reaction, siloxane bonds are formed at the substrate surface, which bonds strongly adhere CAM to the substrate surface. The fluorocarbon group at another site is an important role for anti-stain. As the thickness of CAM is about 1-2 $\mathrm{nm}$, the surface morphology of the substrate can be kept.

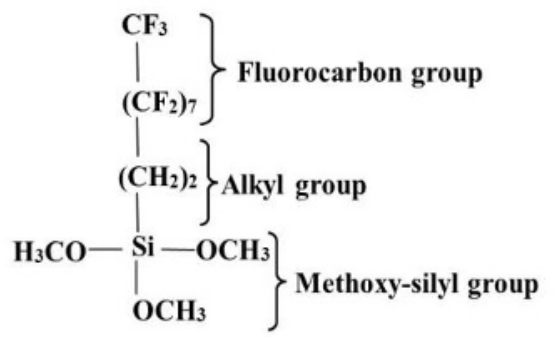

Fig. 5. Molecular formula of the molecule( FAS17) used for CAM, which has a terminal reactive site of the methoxy-silyl group, and a functional site of the fluorocarbon group.

\section{Evaluation methods}

In order to confirm the anti-stain ability of the samples, water drop contact angle measurements were performed at five points on each sample, where water quantity dropped on the sample surface was approximately $3 \mu \mathrm{l}$, and the contact angles calculated by the $\theta / 2$ method. Transmittance of the samples measured from $400 \mathrm{~nm}$ to $780 \mathrm{~nm}$ by using UV-3150 (Shimadzu Corporation). The surface morphology confirmed with field emission type electron beam microscope (FE-SEM: S-900S, Hitachi, Ltd.) at accelerating voltage of $15 \mathrm{kV}$.

\section{Results and discussion}

The average value of the contact angles of 151.8 deg. on sample A is warrants the superhydrophobic surface. However, the average values on other samples are under 150 deg., which means those surfaces are not superhydrophobic. On sample A, we obtained similar superhydrophobicity than that of H.M. Shang's report [6] (Maximum contact angle was 150.0 deg.), where the monolayer was prepared with tridecafluoro-1,1,2,2tetrahydrooctyldimethylchlorosilane (TFCS) on silicabased structure without $\mathrm{ZnO}$ deposition. However, our samples have less contact angle than that of L. Wang's report [7], where the monolayer was prepared with octadecyltrimethoxysilane (OTS) after $\mathrm{SiO}_{2}$ shell deposition on $\mathrm{ZnO}$ nano-wire.

The transmittance spectra are shown in Fig. 6 . Sample B has higher transmittance than $90 \%$, but the average value of the transmittance spectra of the other samples A, C, and D through the measurement wavelength are below $80 \%$. These may depend on the packing density of the $\mathrm{SiO}_{2}$ particles arranged, because the other samples with sparse paced arrangements have lower transmittance, especially in short wavelength range less than $500 \mathrm{~nm}$.

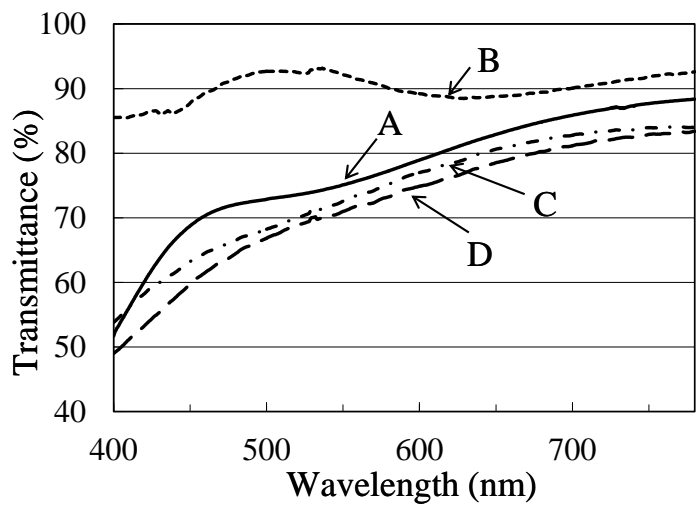

Fig. 6. Transmittance spectra of the samples of visible light range.

Fig. 7 (a)-(d') shows FE-SEM photographs on all samples. On sample A, as shown in fig. 7 (a) and (a'), $\mathrm{ZnO}$ columns have grown surroundingly around the $\mathrm{SiO}_{2}$ particle and raspberry-like structure was formed successfully, but on other samples, such raspberry 
structure were not confirmed, where it seems that $\mathrm{ZnO}$ deposition was occurred just on the top of the $\mathrm{SiO}_{2}$ particle and glass substrate surface.

In fig. 7 (a'), (c'), and (d'), there are some concave areas, which were formed by the sparsity of $\mathrm{SiO}_{2}$ particle arranged. On the other hand, in fig. 7 (b'), sample B has uniform surface with densely packed of $\mathrm{ZnO}$ columns which is due to the dense arrangement of $\mathrm{SiO}_{2}$ particle. Another noticeable feature is pebble lump surface structures on $\mathrm{ZnO}$ columns of sample B and D (fig. 7 (b) and (d)), which is less found on sample C.
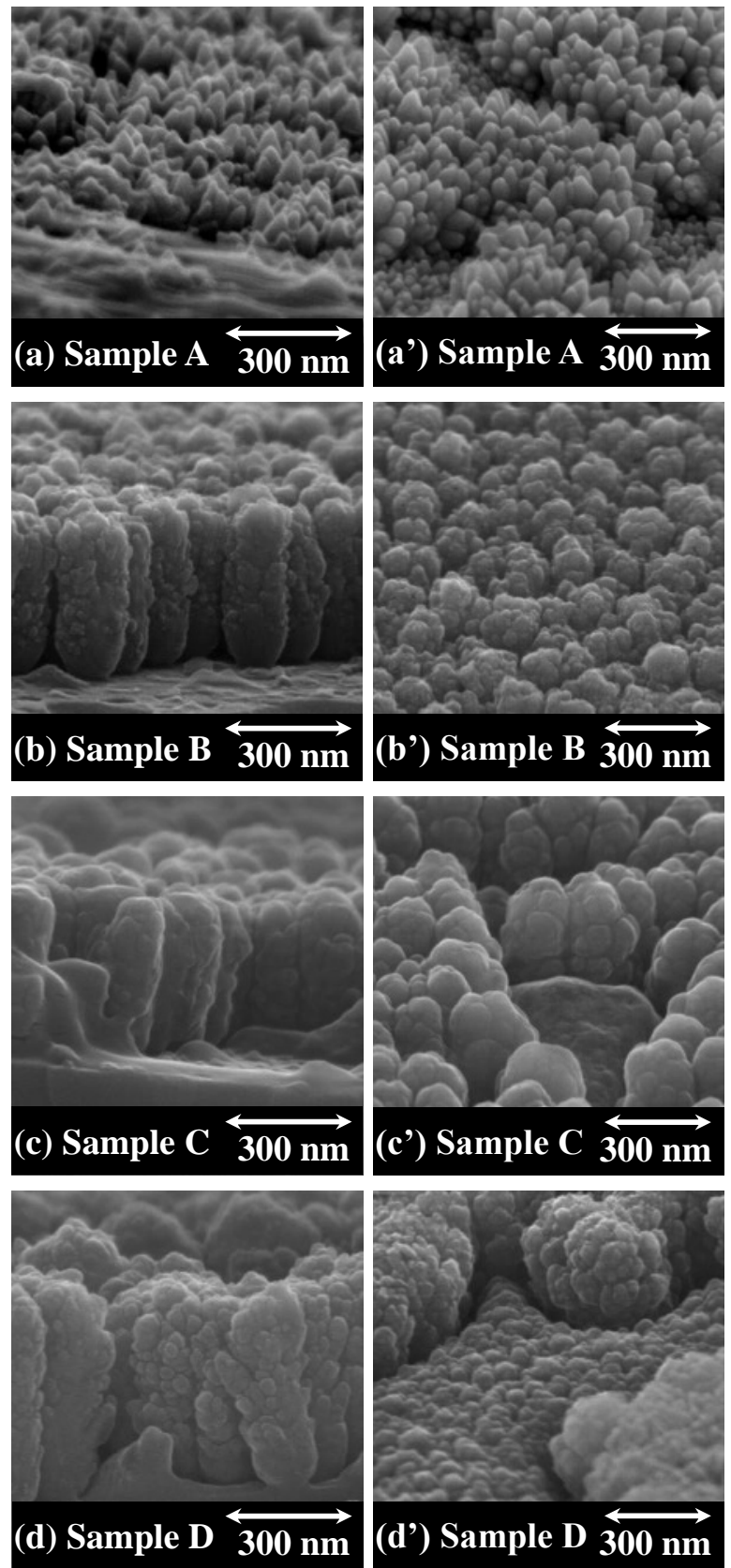

Fig. 7. FE-SEM photographs of the surface structures on sample A, B, C, and D. Images with alphabetical symbol with superscript dash were obtained at another angle for obtaining wider field of view.

There were some differences on the structures between the samples prepared at substrate temperatures of $50^{\circ} \mathrm{C}$ and $215^{\circ} \mathrm{C}$. Those differences may be caused by the substrate temperature during the $\mathrm{ZnO}$ deposition, that is, when the temperature is $215^{\circ} \mathrm{C}, \mathrm{Zn}$ and/or $\mathrm{O}$ atoms deposited on the surface of $\mathrm{SiO}_{2}$ particle can easily move and migrate, and its crystallinity improves. Due to this migration effect, each $\mathrm{ZnO}$ column has sharp edges. Thus sample A has a raspberry-like structure. This uneven surface of sample A showed superhydrophobicity according to Wenzel's or Cassie-Baxter's theory [8]. On the other hand, at the low substrate temperature of $50{ }^{\circ} \mathrm{C}$, the deposited atoms could not migrate, because the thermal energy was insufficient to migrate. As a result, the deposited layer has bad crystallinity, each column was densely packed with the column consisting of relatively rounded $\mathrm{ZnO}$ particles.

\section{Conclusion}

Superhydrophobicity was realized only on sample A having raspberry structure. Although sample B had a high transmittance, hydrophobicity was low. The best anti-stain property will be attained at the condition of pulling speed of $0.23 \mathrm{~mm} / \mathrm{s}$ from the $\mathrm{SiO}_{2}$ particle suspension, and substrate temperature of $215^{\circ} \mathrm{C}$ at the $\mathrm{ZnO}$ deposition. However, in future, we must focus rather lower temperature deposition than $215^{\circ} \mathrm{C}$, because it is needed in order to apply our process to plastic substrates.

\section{Acknowledgement}

This work was supported by the Center for International Exchange at Hanbat National University, Korea, and JSPS KAKENHI Grant Number 22560113, Japan.

\section{References}

1. B. B. Mandelbrot, Fractals: Form, Chance, and Dimension (W.H.Freeman \& Co Ltd, 1977)

2. R. N. Wenzel, Industrial \& Engineering Chemistry, 28, 988 (1936)

3. T. Nishio, M. Meguro, K. Nakamae, M. Matsushita, Y. Ueda, Langmuir 15, 4321 (1999)

4. Y. Suzaki, A. Kawaguchi, T. Murase, T. Yuji, T. Shikama, D. B. Shin, Y. K. Kim, Phys. Status Solidi C 8, 503 (2011)

5. K. Ogawa, T. Ohtake, T. Nomura, M. Soga, N. Mino, Jpn. J. Appl. Phys. 39, 6684 (2000)

6. H. M. Shang, Y. Wang, S. J. Limmer, T.P. Chou, K. Takahashi, G. Z. Cao, Thin Solid Films 472, 37 (2005)

7. L. Wang, X. Zhang, Y. Fu, B. Li, Y. Liu, Langmuir 25, 13619 (2009)

8. A. B. D. Cassie, S. Baxter, Transactions of the Faraday Society 40, 546 (1944) 